\title{
Risco e assistência à dislipidemia no climatério
}

\author{
Risk factors and assistance to dyslipidemia \\ in climacteric women
}

Correspondência:

Sebastiöo Freitas de Medeiros Rua Almirante Henrique Pinheiro Guedes, $s / n$ esquina Major Otávio Pitaluga - Duque de Caxios CEP 78043-306 - Cuiabá/MT

A assistência diferenciada à paciente climatérica deve ter sustentação no conhecimento das condições que, mais freqüentemente, acometem a mulher após os 40 anos de idade, resultando em maior morbiletalidade e repercutindo negativamente na qualidade de vida ao longo do envelhecimento. Há necessidade de harmonizar a longevidade com o viver bem. Identificar e intervir precocemente nas neoplasias mais prevalentes na mulher (colo do útero, mama, pulmão, colo-retal, endométrio e ovários), na osteoporose, alterações no metabolismo glicídico e lipídico e doenças cardiovasculares (DCV) são pilares na qualidade do atendimento ginecológico à mulher climatérica. Entre mulheres, a DCV é a maior causa de morte após a menopausa ${ }^{1,2}$. Logo, definir individualmente o escore de risco para doença ateroesclerótica em dez anos (Framingham), incluindo idade acima de 55 anos, história de doença coronariana em parentes de primeiro grau nas mulheres com menos de 65 anos, presença de insuficiência renal, aumento ventricular esquerdo e síndrome metabólica deve ser atribuição diária do médico no exercício da assistência clínica a esta população.

Estudos epidemiológicos são consistentes na assertiva de que estrogênios ou mesmo estrogênios associados a progestogênios estão relacionados à redução na incidência de $\mathrm{DCV}^{3,4}$. Esta associação explicaria tanto a menor prevalência de DCV nas mulheres antes da menopausa como o aumento desta condição após o estabelecimento do hipoestrogenismo ${ }^{5}$. A plausibilidade biológica do efeito benéfico dos estrogênios sobre o coração e vasos [sujeito 1] e os níveis séricos dos diferentes lipídios [sujeito 2] são robustos. No entanto, estudos clínicos randomizados controlados não confirmaram o benefício esperado sobre as DCV do estrogênio após a menopausa $^{6}$, divergência atribuida ao viés de a estrogenioterapia ter sido iniciada após longos anos de hipoestrogenismo, quando já existiriam alterações no sistema cardiovascular ${ }^{7}$.

Configurando fator de risco das DCV, as modificações nocivas nos lipídios são mais acentuadas após a menopausa, podendo ocorrer elevação nos níveis de triglicerídeos (TG $\geq 150 \mathrm{mg} / \mathrm{dL}$ ), elevações nos níveis de LDL-C (>100 mg/dL) e redução dos níveis de HDL-C $(<40 \mathrm{mg} / \mathrm{dL})$ - condições sabidamente aterogênicas ${ }^{8,9}$. Elevação de $1 \%$ nos níveis de LDL-C aumenta o risco de DCV em $2 \%$, e diminuição de $1 \%$ nos níveis de HDL-C eleva este risco entre 2 e $4,7 \%^{10}$. Seis meses após a menopausa, os níveis de colesterol total (CT), LDL-C e TG elevam-se entre 5 e $10 \%$ e, em dois anos, o HDL-C diminui em aproximadamente $6 \%{ }^{11}$.

Por sorte, dentre os maiores fatores de risco para DCV, a dislipidemia é um fator modificável com intervenções precoces. Assim, é clara a recomendação do seguimento dos 
níveis dos lipídios, tendo-se como meta a manutenção dos níveis de LDL-C abaixo de $100 \mathrm{mg} / \mathrm{dL}$, de HDL-C acima de $60 \mathrm{mg} / \mathrm{dL}$, do CT abaixo de $200 \mathrm{mg} / \mathrm{dL}$ e dos triglicerídeos também abaixo de $100 \mathrm{mg} / \mathrm{dL}^{12}$. Em mulheres brasileiras com idade entre 25 e 45 anos, a prevalência de hipercolesterolemia é de aproximadamente 40\%, havendo níveis mais elevados com o aumento da idade ${ }^{13}$. Estudos em que foram examinadas as elevações isoladas de outros lipídios não são disponíveis. Na prática clínica, observa-se aumento no CT, LDL ou TG, ou diminuição do HDL, tanto de forma isolada, como em várias combinações ${ }^{14}$.

No seu conjunto, as informações mencionadas anteriormente apontam para a relevância da avaliação rotineira dos lipídios na mulher climatérica, principalmente na pós-menopausa. Nesta edição da Revista Brasileira de Ginecologia e Obstetrícia, Oliveira et al. ${ }^{15}$ examinam os fatores de risco associados à dislipidemia em mulheres após a menopausa, incluindo 45 casos (média de idade de 52,1 anos) e 39 controles (média de idade de 52,2 anos), atendidas em ambulatório específico do Sistema Único de Saúde (SUS) em Fortaleza, Ceará. Além das variáveis "idade” e "menopausa”, outros fatores de risco analisados para dislipidemia e comparados entre casos e controles foram "sedentarismo" e "circunferência abdominal maior que 88 cm". Associação estatisticamente significativa foi encontrada apenas em relação a esta variável antropométrica.

A possibilidade de dislipidemia familiar não foi analisada pelos autores, mas mutações em um ou múltiplos genes podem estar envolvidas no metabolismo lipídico, principalmente na hipercolesterolemia isolada. Os autores não mencionaram a prevalência da dislipidemia em mulheres brasileiras com mais de 45 anos - população na faixa etária dos casos e controles estudados. Ausência deste dado limita a estimativa do tamanho da amostra para estudos com este desenho. Mesmo assumindo prevalência de dislipidemia de $40 \%$ nos casos e diferença na ordem de $20 \%$ entre casos e controles, o tamanho mínimo da amostra seria de pelo menos 78 indivíduos em cada grupo, para erro alfa de $5 \%$ e erro beta de $20 \%^{16}$. As afirmativas dos autores de que a dislipidemia no climatério não parece ter relação com alguns fatores determinantes da dislipidemia na população geral e de que a dislipidemia pode ter ocorrido no climatério de modo independente das calorias ingeridas não têm suporte, já que o estudo incluindo um número pequeno de mulheres tem baixo poder. A associação entre razão-cintura-quadril e DCV negada pelos autores foi, na verdade, documentada $(\mathrm{OR}=2,6 ; \mathrm{p}=0,03)$, confirmando resultados de estudos mais robustos.

A maior fraqueza do estudo comentado aqui é o pequeno número de indivíduos incluídos nos dois grupos. O impacto do estudo sobre o tema na prática clínica é pequeno, mas destaca bem a necessidade de mais estudos epidemiológicos nessa população, neste tópico, no país. Os autores recomendam acertadamente a verificação dos lipídios na rotina da assistência à mulher pós-menopausa em todas as esferas da atividade médica no país. Há que se desenhar estudos focando a nossa realidade e os nossos costumes. Assim, será possível fazer análise comparativa com outros países e propor medidas corretas aos dirigentes do sistema de Saúde brasileiro. Pela dimensão do impacto da hiperlipidemia na DCV em todo mundo, é importante que qualquer profissional de Saúde tenha conhecimento das recomendações atuais para rastreamento dos lipídios ${ }^{11}$. Os ginecologistas, em particular, sendo clínicos da mulher, devem garantir que todos os fatores de risco para DCV sejam focados na rotina de suas ações assistencialistas. Na verdade, o ginecologista deve assistir a mulher nesta parte da vida com visão ampla, incluindo tópicos como a educação para atividade física individualizada e regular, educação dietética e, eventualmente, terapia hormonal - de acordo com as indicações aceitas atualmente, balanceada com a diminuição gradual da produção ovariana dos esteróides sexuais.

\section{Referências}

1. Bittner V. Women and coronary heart disease risk factors. J Cardiovasc Risk. 2002;9(6):315-22.

2. Schmitt ACB, Cardoso MRA, Aldrighi JM. Tendências da mortalidade em mulheres brasileiras no climatério. Rev Bras Crescimento Desenvolv Hum. 2008; 18(11):11-5.

3. Grodstein F, Manson JE, Colditz GA, Willett WC, Speizer FE, Stampfer M. A prospective, observational study of postmenopausal hormone therapy and primary prevention of cardiovascular disease. Ann Intern Med. 2000;133(12):933-41.

4. Stampter M, Colditz GA, Willett WC, Manson JE, Rosner B, Speizer FE, et al. Postmenopausal estrogen therapy and cardiovascular disease. Ten-year follow-up from the nurses' health study. N Engl J Med. 1991;325(1 1):756-62.

5. Mobasseri S, Liebson PR, Klein LW. Hormone therapy and selective estrogen receptor modulators for prevention of coronary heart disease in postmenopausal women estrogen replacement from the cardiologist's perspective. Cardiol Rev. 2004;12(6):287-98.

6. Manson JE, Bassuk SS. Invited commentary: hormone therapy and risk of coronary heart disease. Why renew the focus on the early years of menopause? Am J Epidemiol. 2007;166(5):511-7. 
7. Cho GJ, Lee JH, Park HT, Shin JH, Hong SC, Kim T, et al. Postmenopausal status according to years since menopause as an independent risk factor for the metabolic syndrome. Menopause. 2008;15(3):524-9.

8. Austin MA. Plasma triglyceride as a risk factor for coronary heart disease. The epidemiologic evidence and beyond. Am J Epidemiol. $1989 ; 129(2): 249-59$.

9. Verhoeven MO, van der Mooren M, Teerlink T, Verheijen RH, Scheffer PG, Kenemans P. The influence of physiological and surgical menopause on coronary heart disease risk markers. Menopause. 2008;16(1):1-12.

10. Manson JE, Tosteson H, Ridker PM, Satterfield S, Herbert P, O'Connor GT, et al. The primary prevention of myocardial infarction. N Engl J Med. 1992;326(21):1406-16.

11. Jensen J, Nilas L, Christiansen C. Influence of menopause on serum lipids and lipoproteins. Maturitas. 1990;12(4):321-31.

12. Mosca, L, Banka CL, Benjamin EJ, Berra K, Bushnell C, Dolar R, et al. Evidence-based guidelines for cardiovascular disease prevention in women: 2007 update. J Am Coll Cardiol. 2007;49(1 1):1230-50.

13. Sposito AC, Caramelli B, Fonseca FA, Bertolami MC, Afiune Neto A, Souza AD, et al. IV Diretriz Brasileira sobre Dislipidemias e Prevenção da Aterosclerose: Departamento de Aterosclerose da Sociedade Brasileira de Cardiologia. Arq Bras Cardiol. 2007;88 Supl 1:2-19.

14. Schnatz PF, Schnatz JD. Dyslipidemia in menopause: mechanisms and management. Obstet Gynecol Surv. 2006;61(9):608-13.

15. Oliveira TR, Sampaio HAC, Carvalho FHC, Lima JWO. Fatores associados à dislipidemia na pós-menopausa. Rev Bras Ginecol Obstet. 2008; 30(12):594-601.

16. Daly LE, Bourke GJ. Interpretation and uses of medical statistics. London: Wiley-Blackwell; 2000. p. 269-308. 